\title{
To grip or not to grip
}

Optical feedback enables a lightresponsive smart material to selectively detect and automatically grip specific objects, report Owies Wani, Hao Zeng and Arri Priimagi in Nature Communications.

Smart materials that autonomously respond to changes in their environment have recently garnered interest in the burgeoning area of biomimetic microrobotics. Although such materials may deform when the chemical composition or light levels around them change, they cannot distinguish between objects or adjust their response to different triggers. Inspired by a carnivorous plant, the Venus flytrap,

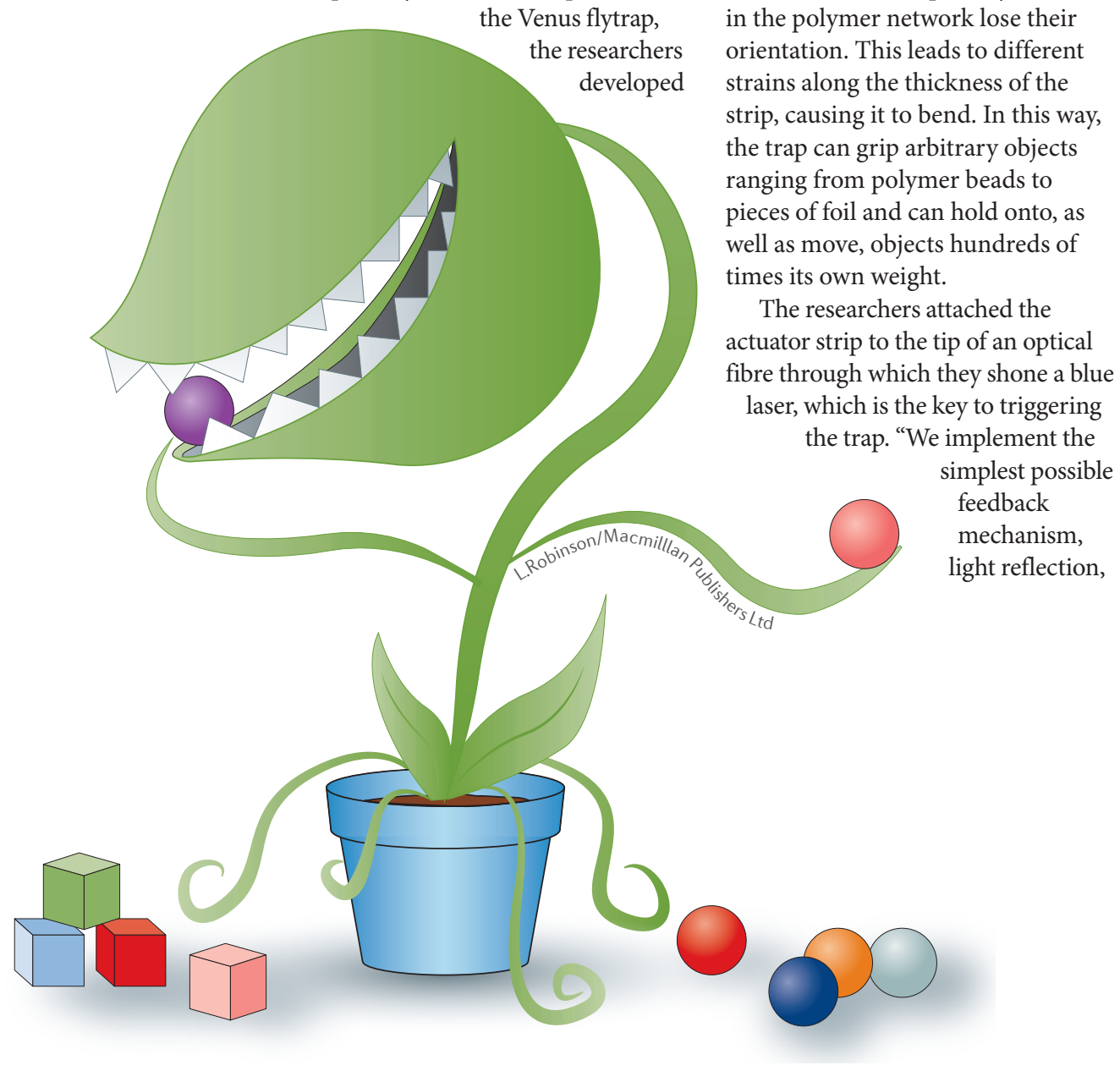

a smart material system that uses light to achieve this goal. "Whereas the snapping motion of the Venus flytrap, which leads to the fast capture of small insects, has been studied quite a bit, the fact that it can also distinguish insects and other prey from random dust by sensing their mechanical triggers has received less attention," says Zeng.

Their artificial flytrap is made from a thin strip of liquid-crystal elastomer doped with dye molecules and, unlike its biological model, it responds to light rather than to a mechanical trigger: when light hits this material, the liquid crystals in the polymer network lose their orientation. This leads to different of the strip, causing it to bend. In this way, the trap can grip arbitrary objects ranging from polymer beads to pieces of foil and can hold onto, as well as move, objects hundreds of mes its own weight.

The researchers attached the actuator strip to the tip of an optical fibre through which they shone a blue which is the key to triggering implement the implest possible feedback mechanism, ght reflection, to obtain light-induced bending," explains Zeng. In other words, when an object enters the light cone above the trap, it reflects some of the laser light back onto the elastomer strip, which bends in response.

Although this may at first sound like an ordinary light barrier, the feedback mechanism is more sophisticated, with the exact reaction of the liquid-crystal network depending on the intensity of light and thus on the amount and direction of light reflected by the object in the beam. The researchers tested the selectivity of their trap with various objects that scatter and absorb light differently and found that the minimum laser intensity necessary to close the trap and the closing speed are specific to each object. They showed that the trap can automatically select a strongly scattering cube when it passes through the field of view, whereas less reflecting cubes are left untouched. The team is "very excited about this result, which demonstrates that small-sized actuators are capable of 'big decisions"' remarks Priimagi.

The ability to autonomously recognize and respond to a certain trigger brings biomimetic microrobots one step closer to full automation and also to their model organisms. Therefore, improving selectivity is one of the main goals for future research in this direction. "We are working towards making the flytrap 'smarter' and more functional, such that it could recognize and distinguish between objects of different colours," concludes Priimagi.

Nina Meinzer Associate Editor, Nature Communications 